\section{BMJ Global Health}

\title{
Associations of women's empowerment with neonatal, infant and under-5 mortality in low- and /middle-income countries: meta-analysis of individual participant data from 59 countries
}

\author{
David T Doku, ${ }^{1,2}$ Zulfiqar A Bhutta, ${ }^{3,4}$ Subas Neupane ${ }^{2}$
}

\begin{abstract}
To cite: Doku DT, Bhutta ZA, Neupane S. Associations of women's empowerment with neonatal, infant and under-5 mortality in low- and /middle-income countries: meta-analysis of individual participant data from 59 countries. BMJ Global Health 2020;5:e001558. doi:10.1136/ bmjgh-2019-001558
\end{abstract}

Handling editor Dr Sanni Yaya

- Additional material is published online only. To view please visit the journal online (http://dx.doi.org/10.1136/ bmjgh-2019-001558).

Received 11 March 2019 Revised 25 June 2019 Accepted 29 June 2019

Check for updates

\section{(c) Author(s) (or their} employer(s)) 2020. Re-use permitted under CC BY-NC. No commercial re-use. See rights and permissions. Published by BMJ.

For numbered affiliations see end of article.

\section{Correspondence to}

Dr David T Doku, Department of Population and Health, University of Cape Coast, Cape Coast, Ghana;

dokudavid@gmail.com

\section{ABSTRACT}

Background Child survival and women's empowerment are global public health concerns and important sustainable development goals (SDGs). Low- and middle-income countries (LMICs) have the largest burden of both phenomena. The aim of this study is to investigate a measure of women's empowerment at individual and population levels and its potential associations with neonatal, infant and under-5 mortality at national and regional levels in 59 LMICs. Methods We used pooled population-based crosssectional surveys from 59 LMICs $(n=612529)$ conducted from 2000 to 2015 using standardised protocols. We constructed individual-level women's empowerment index (ILWEI) and population-level women's empowerment index (PLWEI) for LMICs and investigated the potential associations of these measures with neonatal, infant and under-5 mortality using two-stage random-effect individual participant data (IPD) meta-analysis.

Results The pooled neonatal mortality rate was 24 per 1000 live births. Infant and under-5 mortality rates were 43 and $55 / 1000$ live births, respectively. In the pooled sample, $61.6 \%$ and $19.9 \%$ of women had autonomy regarding their healthcare and household decision-making, respectively, whereas $56.0 \%$ rejected domestic violence against women for any reason. IPD meta-analysis showed that children of women with low ILWEl had a higher risk of neonatal (OR: $1.18,95 \% \mathrm{Cl} 1.14$ to 1.22$)$, infant (OR: $1.12,95 \% \mathrm{Cl} 1.08$ to $1.17)$ and under-5 (OR: $1.12,95 \% \mathrm{Cl} 1.07$ to 1.18 ) mortality compared with children of high ILWEI. Similar relationships were found across most of the regions as well as between PLWEl and all the three outcomes.

Conclusions Women's empowerment at individual and population levels is associated with neonatal, infant and under-5 mortality in LMICs. Our study underscores the importance of women's empowerment in accelerating progress towards the attainment of the SDG targets for child survival in LMICs. Multi-sectoral and concerted efforts are necessary to eliminate preventable child mortality in these countries.

\section{INTRODUCTION}

The burden of neonatal, infant and under-5 mortality in low- and middle-income countries

\section{Key messages}

What is already known?

- Women's empowerment is a key global developmental goal (Sustainable Development Goal 5 (SDG 5)) as well as a means to advance the other SDGs, including those related to neonatal, infant and under-5 survival (SDG 3). However, there is no standard or consistent measure for women's empowerment across low/middle-income countries (LMICs) and diverse contexts.

What are the new findings?

- Women's empowerment was relatively low in LMICs.

- Children of women with low empowerment at both individual and population levels had a higher risk of neonatal, infant and under-5 mortality. Individual participant data meta-analysis shows evidence of associations of individual-level women's empowerment index with neonatal, infant and under-5 mortality in most countries and in every region in LMICs.

- Our findings suggest that women's empowerment is critical to achieving SDG 3, which aims at reducing neonatal mortality to 12 deaths per 1000 live births and under-5 mortality to 25 death per 1000 live births by 2030 .

What do the new findings imply?

- Healthcare systems in LMICs should be structured to reach less empowered women's children with lifesaving interventions.

- Our findings further underscore the need for multisectoral and concerted efforts to eliminate preventable child mortality in LMICs as well as making progress towards achieving the SDGs.

(LMICs) remains high despite significant progress in recent decades, especially the Millennium Development Goal (MDG) 4 target of two-thirds reduction in the under- 5 mortality between 1990 and $2015 .{ }^{1-9}$ Only 24 out of the 139 LMICs met the MDG 4. ${ }^{4-6}$ Under-5 mortality ratio is 1 in 147 in 
high-income countries, whereas in sub-Saharan Africa and Southern Asia, the ratio is 1 in 12 and 1 in 19, respectively. The Sustainable Development Goal (SDG) 3, which aims at reducing neonatal mortality to 12 deaths per 1000 live births and under-5 mortality to 25 deaths per 1000 live births by 2030 is a global commitment to tackle the unfinished work of the MDG $4 .^{10}$

Efforts to address child mortality have rightly focused on tackling immediate causes such as intrapartum-related events, pneumonia, sepsis and diarrhoea. ${ }^{8}$ However, relatively distal factors, such as gender equality and women's empowerment that fuel the immediate causes, have received little attention. ${ }^{11}$

Women's empowerment is conceptualised as the process of enabling women with less influence or power to be able to make informed choices in all matters that affect their health and well-being. Empowerment entails two important constructs: the ability to formulate one's own choices and act on them (agency) and the means through which one's choices are exercised (resources). ${ }^{12-14}$ Gender equality and women's empowerment are important components of the global developmental agenda. Efforts towards MDG 3, which sought to promote gender equality and women's empowerment, did lead to some significant gains over the last two decades. ${ }^{4}$ However, girls and women still face many social, economic and cultural barriers, which hinder their own perception of their roles as well as the ability to achieve them. ${ }^{15}$ Given the importance of gender equality and women's empowerment for social, economic and sustainable development, a separate goal, SDG 5 now underscores the global commitment towards addressing these issues. ${ }^{10}$ The SDGs are considered as an indivisible whole. Therefore, such a gap has important implications for their overall attainment. ${ }^{1017}$

Women with low agency and resources may be limited in their ability to make choices regarding their own health and those of their children. Consequently, these circumstances can contribute to their own ill health and risk of mortality as well as that of their children. ${ }^{16}{ }^{18}$ In two recent systematic reviews, one found an inverse association of women's empowerment with child undernutrition in South Asia ${ }^{19}$ and the other a positive association of women's empowerment with completion of childhood immunisation in LMICs. ${ }^{20}$ Likewise, lack of women's empowerment and participation in community healthcare programmes were implicated in the failure of some countries to achieve the MDGs 4 and 5 targets for reduction in maternal and child mortality. ${ }^{21}$ James-Hawkins $e t$ al in their systematic review of the relationship between women's agency and current contraceptive use in LMICs found a positive association. ${ }^{22}$ Although both child survival and women's empowerment are of global importance, our systematic review of the literature suggests that no study has used meta-analysis of individual participant data (IPD) to explicitly investigate the effect of women's empowerment on child survival outcomes in LMICs, the countries with the highest burden of both phenomena. ${ }^{4-6} 21$
Earlier researchers have used a number of context and region-specific indices of women's empowerment. For instance, Ewerling et $a t^{23}$ constructed Survey-based Women's emPowERment index (SWPER) as a tool for the assessment of women's empowerment in sub-Saharan Africa. SWPER was developed for sub-Saharan Africa and its applicability to other regions, notably Latin America and the Caribbean has been questioned. Akseer $e t a l^{24}$ also used different indicators as measures of empowerment for women in the Islamic world. These various indicators underscore the multidimensional nature of the construct of women's empowerment. We used unique individual-level data, which were collected using standardised demographic and health surveys (DHSs) and population-level data to investigate the effect of women's empowerment on neonatal, infant and under-5 mortality in 59 LMICs. We also aimed to investigate the regional differences in these associations.

\section{METHODS \\ Data}

We used nationally representative cross-sectional data from 59 LMICs collected in DHSs using standardised questionnaires and protocols in order to facilitate international comparison. The DHSs have generated highquality data on important demographic, economic, social and health for LMICs and have been used in high-quality research. The DHS data were collected at about 5-year intervals across LMICs. DHSs collect data by interviewing women of reproductive age (15-49 years), their children and their households. DHSs are available to investigators through the World Wide Web (http://www.dhsprogram. com). Briefly, DHSs used a stratified two-stage random sampling approach, consisting of a selection of census enumeration areas based on a probability, followed by a random selection of household from a complete listing of a household within the selected enumeration areas. In all the 59 countries, DHSs followed the same standard procedures. Further descriptions of DHS sampling procedures, validation of questionnaires and data collection methods are published elsewhere (http://www.dhsprogram.com). In this study, we used data collected on the outcome of the most recent live birth within 5 years preceding the survey for each woman of reproductive age 15-49 years. We used the most recent publicly available datasets conducted between 2000 and 2015 to generate a large dataset with sufficient statistical power to investigate the effect of women's empowerment on neonatal, infant and under- 5 mortality. Our study data represent $42 \%$ of the 139 LMICs globally. We restricted our analyses to the most recent singleton live birth for each woman of reproductive age $(n=612529)$. The countries were divided into three income groups according to the World Bank list of economies (24 low-income countries, 24 lower-middleincome countries and 11 upper-middle-income countries $^{25}$ and their regional classifications according to the WHO (sub-Saharan, East Asia and Pacific, Europe and 
Central Asia, Latin America and Caribbean, Middle East and North Africa, and South Asia) (table 1).

\section{Ethical clearance}

Ethical clearance for the respective surveys was granted in the respective countries and permission to analyse the data was obtained from the DHS programme (www. dhsprogram.com).

\section{Patient Involvement Statement}

Patients were not involved in the design and implementation of this research.

\section{Outcome measurements}

The outcome measures were neonatal, infant and under-5 mortality. Under-5 mortality was defined as the death of a live-born baby within 5 years of life. Infant mortality was defined as the death of live-born baby within 12 months of life and neonatal death refers to the death of a live-born baby within the first 28 days of life. These were estimated based on information on the date of birth, date of death and the survival status, which were collected at the time of the survey through interviews. Age at death was recorded in days if the child died within 28 days of birth, otherwise in months.

\section{Measures of women's empowerment}

In this study, we used indicators, which are applicable across a wide range of LMICs and which reflect the multidimensionality of the concept of empowerment to construct women's empowerment indices. We developed two indices, individual-level women's empowerment index (ILWEI) and population-level women's empowerment index (PLWEI) to measure women's empowerment. The ILWEI was measured using three major indicators of women's autonomy variables, namely autonomy in healthcare decision making, household decision making (making large household purchases, visits to family or relatives and how to spend money), and justification of domestic violence against women (if she goes out without telling her husband, neglects the children, argues with her husband or if she refuses to have sex with her husband) and three social independence variables, namely women's education, frequency of reading newspaper and respondents who worked during past 12 months (online supplementary table $\mathrm{S} 1$ ). We constructed the ILWEI based on women's responses to the three measures of autonomy and other three variables on social independence and categorised the scores into terciles as follows: low, medium and high. Furthermore, we constructed PLWEI based on three populationlevel indicators, namely the proportion of the female population with at least secondary education, the proportion of females' share of parliamentary seats and the female labour force participation rates and categorised this into terciles as low, medium and high. These indicators were adapted from the United Nations Development Programme's measure of the ratio of female to male human development index. ${ }^{26}$
Background characteristics of the mother (age, wealth quintile, parity and partner's education) were also included in the analysis as covariates consistent with the previous study. ${ }^{9}$ The wealth quintile is the composite measure of the household's cumulative living standard based on ownership of specified assets categorised into quintiles: poorest, poorer, middle, richer and richest. Body mass index (BMI) was calculated based on the height and weight of women, which were measured during the survey. BMI is used as a continuous variable in the analysis.

BMI was calculated based on the height and weight measurement of women and used as a continuous variable.

\section{Statistical analysis}

Data editing and imputation of missing procedures were conducted by the DHS Programme before data were released for use. ${ }^{27}$ The DHSs in LMICs are prone to incomplete, partial or inconsistent reporting of response for different questions because of its complex questionnaires. The purpose of data editing and imputation in the DHSs is to accurately reflect the population studied and make it useable for analysis. ${ }^{27}$ We further conducted data auditing and validation for each country using the countries' survey protocols. Population sample weights were applied to the pooled data (table 1). Under-5 mortality per 1000 live births and the proportion of women who had no ILWEI in each country are presented in the world map (figure 1A,B). We conducted a principal component analysis (PCA) to construct ILWEI and PLWEI separately at individual and population levels, respectively, on a pooled dataset (online supplementary tables S2 and S3). We ranked the countries according to the level of women's empowerment based on the PCA scores of ILWEI and PLWEI. We used two-stage randomeffects IPD meta-analysis. First, we used multivariable logistic regression analysis to estimate ORs and their $95 \%$ CIs to investigate the association of neonatal, infant and under-5 mortality with ILWEI for each country and region, adjusting for the sociodemographic factors. We also adjusted the models for the effect of the multistage cluster sampling. Second, we conducted a meta-analysis of those ORs from the logistic regression analysis to investigate the effect of women's empowerment on neonatal, infant and under-5 mortality for each country and region using random-effects meta-analysis tools in Stata. The estimates for dummy women's empowerment index variable (low and medium combined as low vs high) were analysed and plotted for each country and region. The true effect of the independent variable in each country is randomly distributed between countries with variance $\tau^{2}$.

The estimate of the between-country variance $\tau^{2}$ modifies the weights used to calculate the pooled estimate. A random effect is given by: $O R_{R}=\frac{\sum_{i=1}^{k} w i O R_{i}}{\sum_{i}^{k} w i}$. Where $w i=\frac{1}{v_{i}+\tau^{2}}$ and the heterogeneity is estimated by formula $\mathrm{Q}=\sum_{i=1}^{n}\left\{\left(\frac{1}{\text { var }}\right) x(\text { individual effect }- \text { pooled effect })^{2}\right\}$. 
Table 1 Distribution of women's empowerment measures by countries and regions

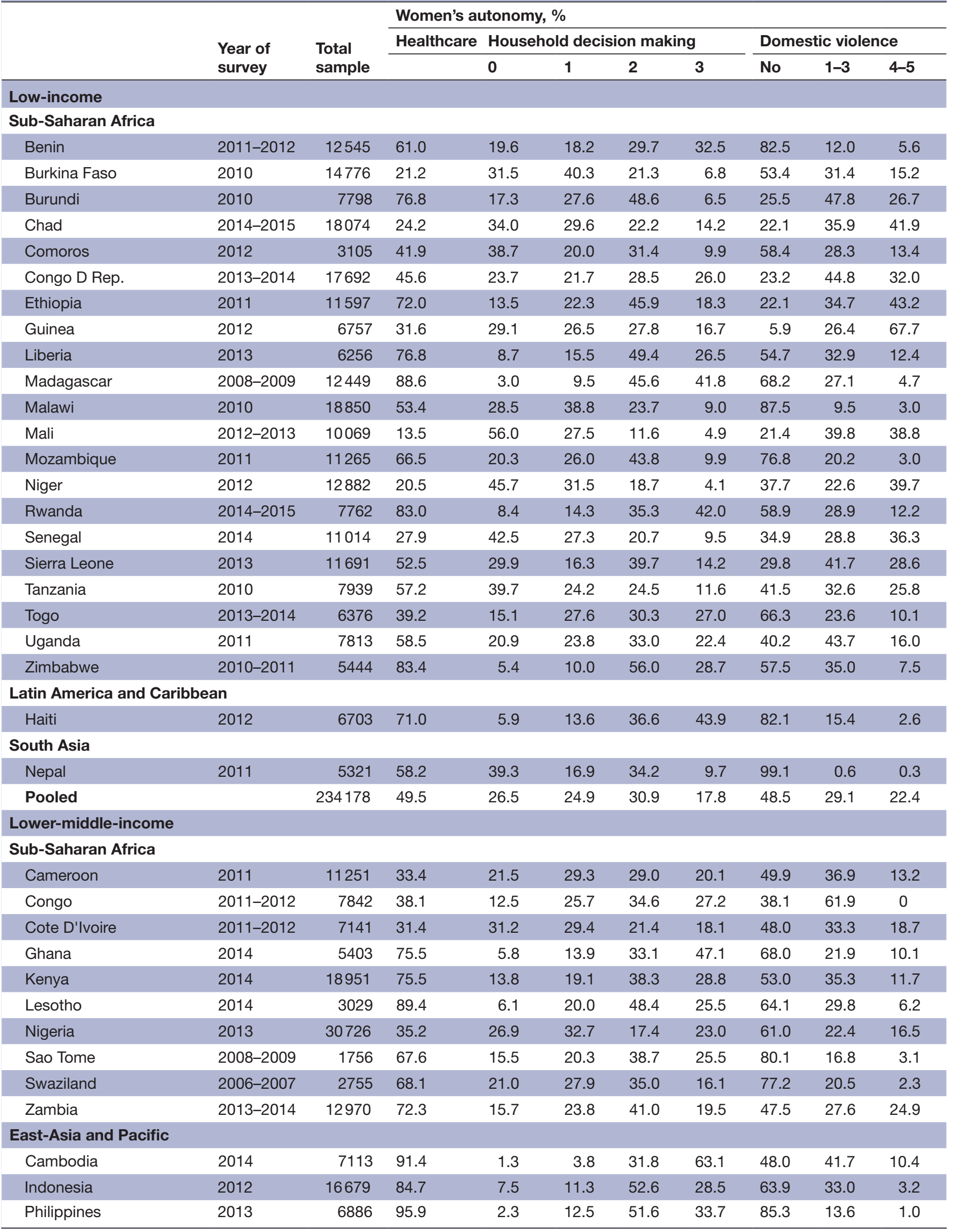


Table 1 Continued

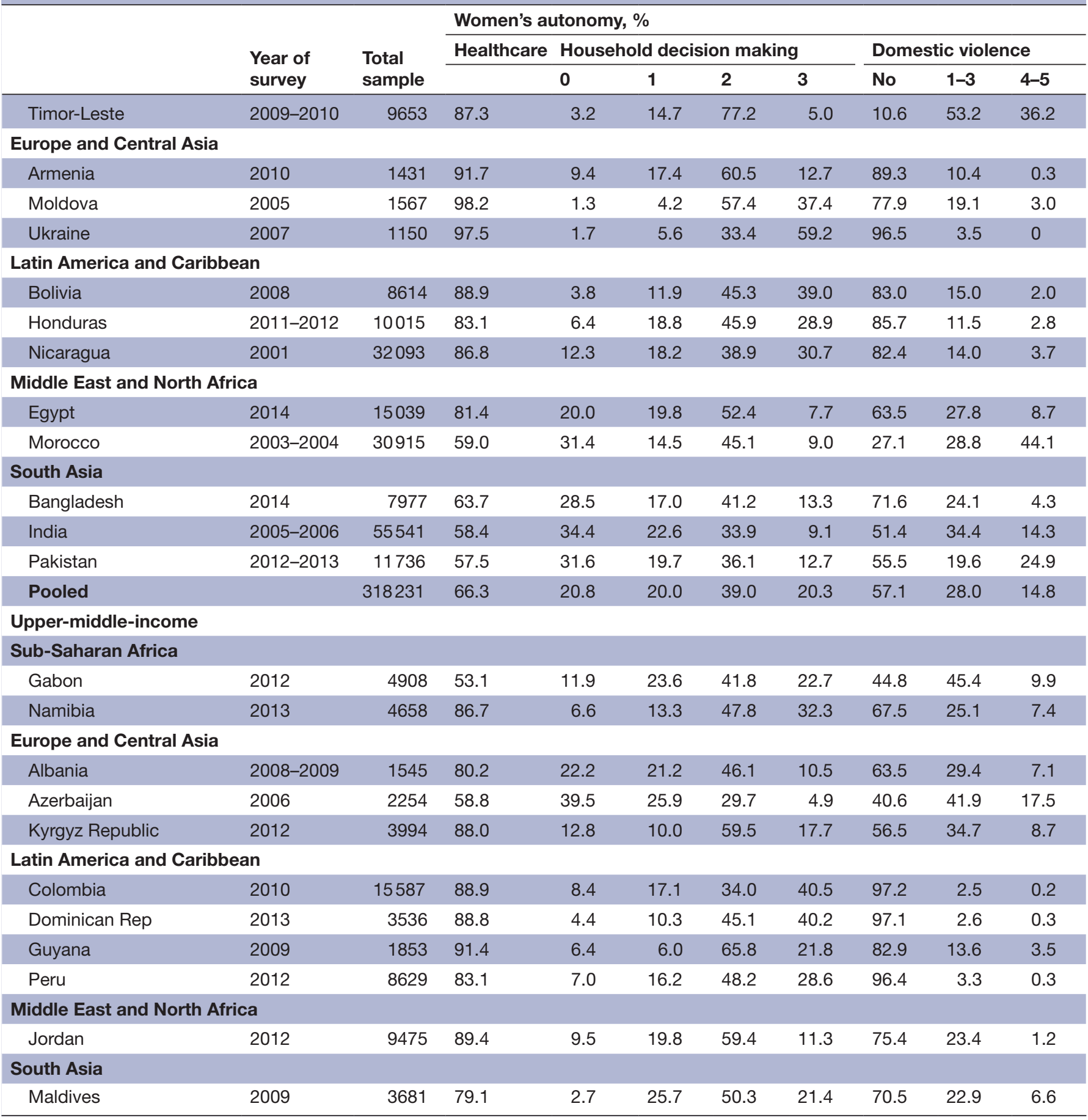

Where var $=\left\{\frac{(\text { upper } C I-\text { lower } C I)}{2 x z}\right\}^{2}$.

The associations of PLWEI with neonatal, infant and under-5 mortality by regions (low income, lower-middleincome and upper-middle-income) were presented in scatter plots. For the scatter plots continuous scores of PLWEI were used. We also conducted a sensitivity analysis of the associations of our ILWEI with neonatal, infant and under-5 mortality in comparison with SWPER ${ }^{24}$ (online supplementary table S5, online supplementary figures S1-S3). All statistical analyses were conducted using STATA/SE V.14.0.

\section{RESULTS}

The distribution of women's empowerment-related measures by income levels of the countries and the regions are presented in table 1 . In the pooled sample, $61.6 \%$ of women had autonomy in decision making regarding their healthcare, $19.9 \%$ had autonomy in all the three indicators of household decision making and $56.0 \%$ 

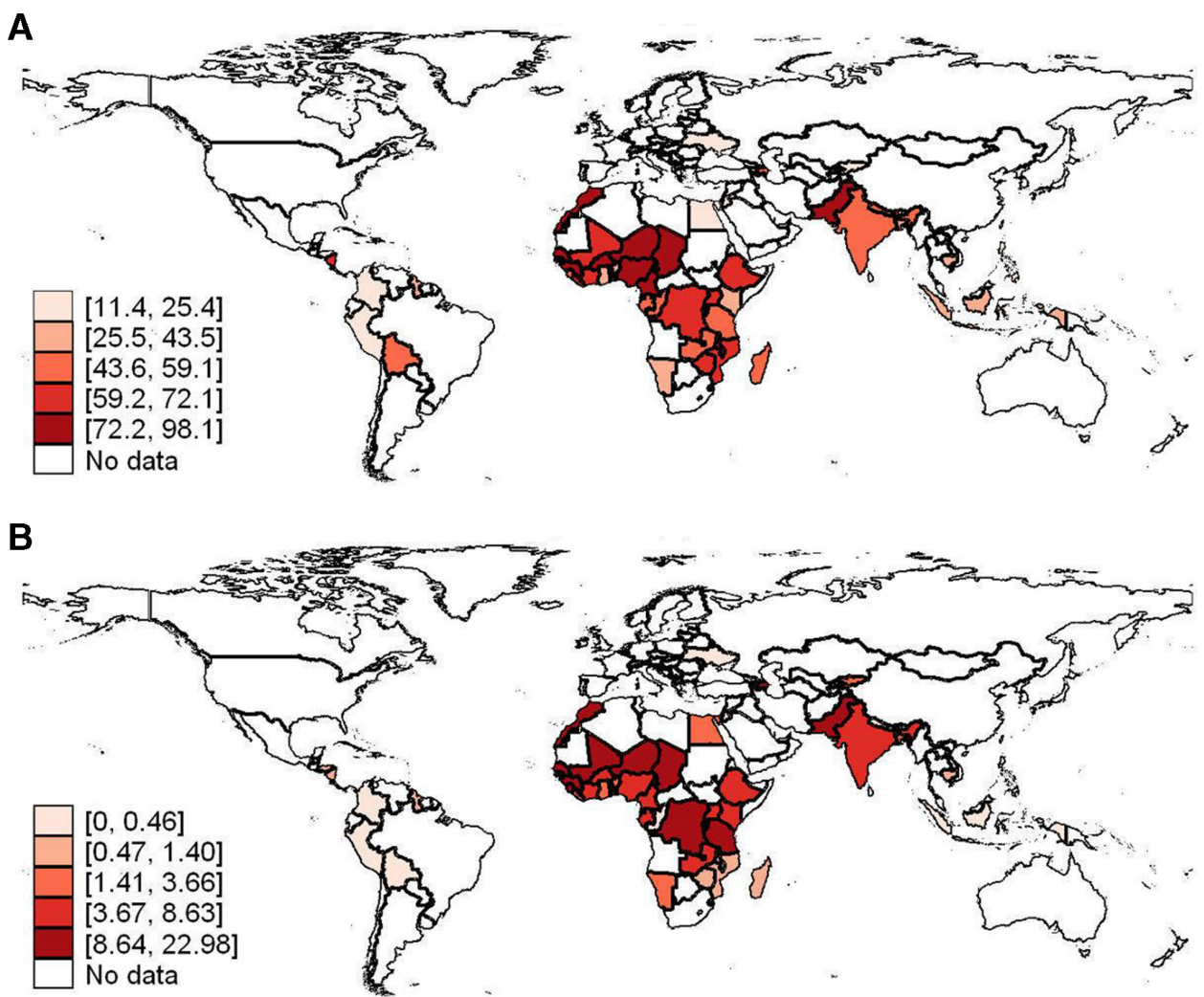

Figure 1 World MAP showing the $(\mathrm{A})$ proportion of under-5 mortality per 1000 live births and (B) proportion of women without empowerment in all three indicators (autonomy in healthcare decision making, household decision making and rejecting domestic violence against women for any reason).

rejected domestic violence against women for any reason. Country-specific under-5 mortality ranged from 11 per 1000 live births in Moldova to 98 per 1000 live births in Senegal (figure 1A). The proportion of women without empowerment in all three indicators varies from 0 in Armenia and Colombia to $22.98 \%$ in Guinea (figure 1B).

The level of women's empowerment varies across countries (online supplementary table S4). By ILWEI, Ukraine (1.64), Armenia (1.51) and Moldova (1.51) had the highest empowerment scores, whereas Guinea $(-1.19)$, Mali $(-0.90)$ and Burkina Faso $(-0.89)$ had the lowest scores. The level of empowerment by PLWEI ranges from the lowest -1.79 (Niger) to highest 2.38 (Moldova).

A meta-analysis of country-level associations of ILWEI with the child mortality outcomes, adjusted for the sociodemographic factors, are presented in figure 2A-C. This analysis shows minimal heterogeneity and suggests that in most countries, children of women with low empowerment were more likely to die as neonates, infants or before their fifth birthdays. The strongest association of women's empowerment with neonatal mortality was found in Dominican Republic (OR: 3.51, CI 1.12 to 10.92); the association with infant mortality was strongest in Dominican Republic (OR: 2.74, 1.07$6.97)$, whereas the association with under-5 mortality was strongest in Dominican Republic (OR: 3.18, CI 1.32 to 7.66). Nevertheless, there were variations in the associations of women empowerment with the three child mortality outcomes across the countries. In some countries, low ILWEI was associated with a lower risk of infant, under-5 and child mortality compared with high ILWEI, whereas in other cases, the associations not statistically significant.

The meta-analysis of the effect of low ILWEI on neonatal, infant and under-5 mortality showed clear and consistent associations for all the three-mortality outcomes (figure 3A-C). Low ILWEI status of a woman increased the risk of the child dying within the first 28 days of life (OR: $1.18,95 \%$ CI 1.14 to 1.22 ), before the first birthday (OR 1.12, 95\% CI 1.08 to 1.17) or before the fifth birthday (OR: $1.12,95 \%$ CI 1.07 to 1.18 ) compared with the child of a woman with high ILWEI. However, there were variations in the associations of women empowerment with the three child mortality outcomes across the regions. In most regions, low ILWEI was associated with a higher risk of infant, under-5 and child mortality compared with high ILWEI. On the other hand, in a few regions, low ILWEI was associated with a lower risk of infant, under-5 and child mortality compared with high ILWEI, whereas in other cases, the associations were not statistically significant. Furthermore, there was moderate or no heterogeneity in the effects across regions.

Scatter plots of the correlation of PLWEI are presented in figure 4A-C. Overall, the higher the empowerment of a woman the less likelihood of her child dying as a neonate, an infant or a child under-5 years of age (figure $4 \mathrm{~A}-\mathrm{C}$ ). 
A

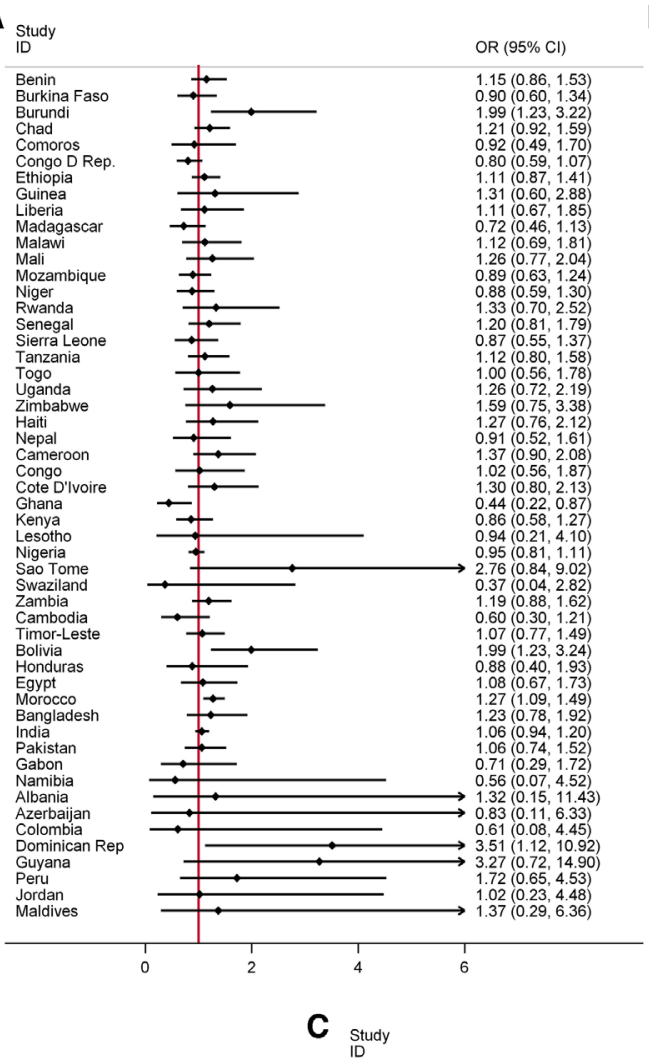

B
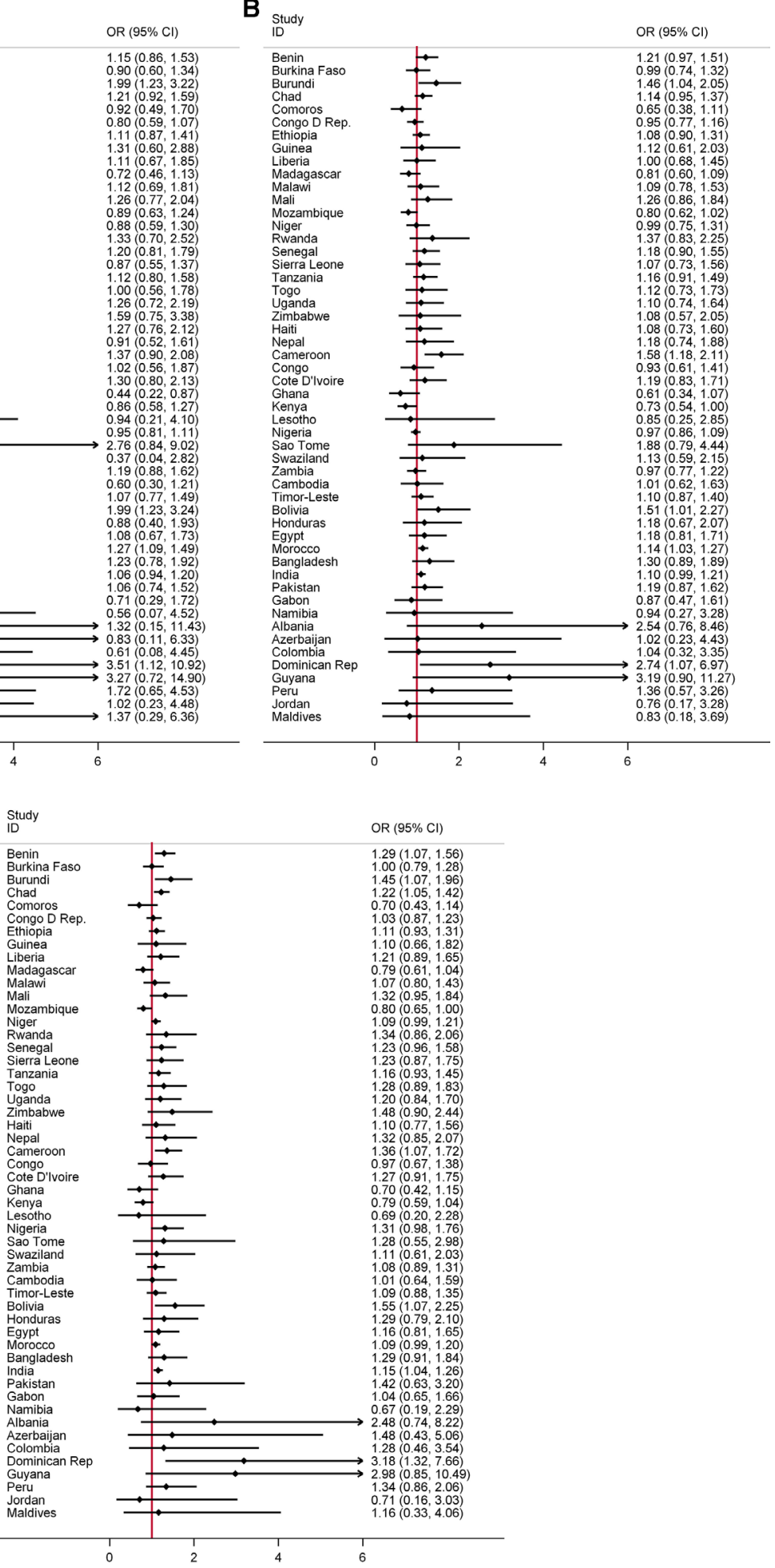

Figure 2 (A) Meta-analysis of the association of low ILWEI with neonatal mortality. ORs and their 95\% Cls adjusted for maternal age, wealth quintile, BMI, parity and partner's education. Seven countries (Armenia, Indonesia, Kyrgyz Republic, Moldova, Philippines, Ukraine and Nicaragua) were excluded in the analysis due to fewer or no cases of neonatal mortality per the categories of the independent variable (low ILWEI). (B) Meta-analysis of the association of low ILWEI with infant mortality. ORs and their $95 \%$ Cls adjusted for maternal age, wealth quintile, BMI, parity and partner's education. Seven countries (Armenia, Indonesia, Kyrgyz Republic, Moldova, Philippines, Ukraine and Nicaragua) were excluded in the analysis due to fewer or no cases of infant mortality per the categories of the independent variable (Iow ILWEI). (C) Meta-analysis of the association of low ILWEI with under-5 mortality. ORs and their $95 \%$ Cls adjusted for maternal age, wealth quintile, BMI, parity and partner's education. Seven countries (Armenia, Indonesia, Kyrgyz Republic, Moldova, Philippines, Ukraine and Nicaragua) were excluded in the analysis due to fewer or no cases of under-5 mortality per the categories of the independent variable (low ILWEI). BMI, body mass index; ILWEI, individual-level women's empowerment index. 
A

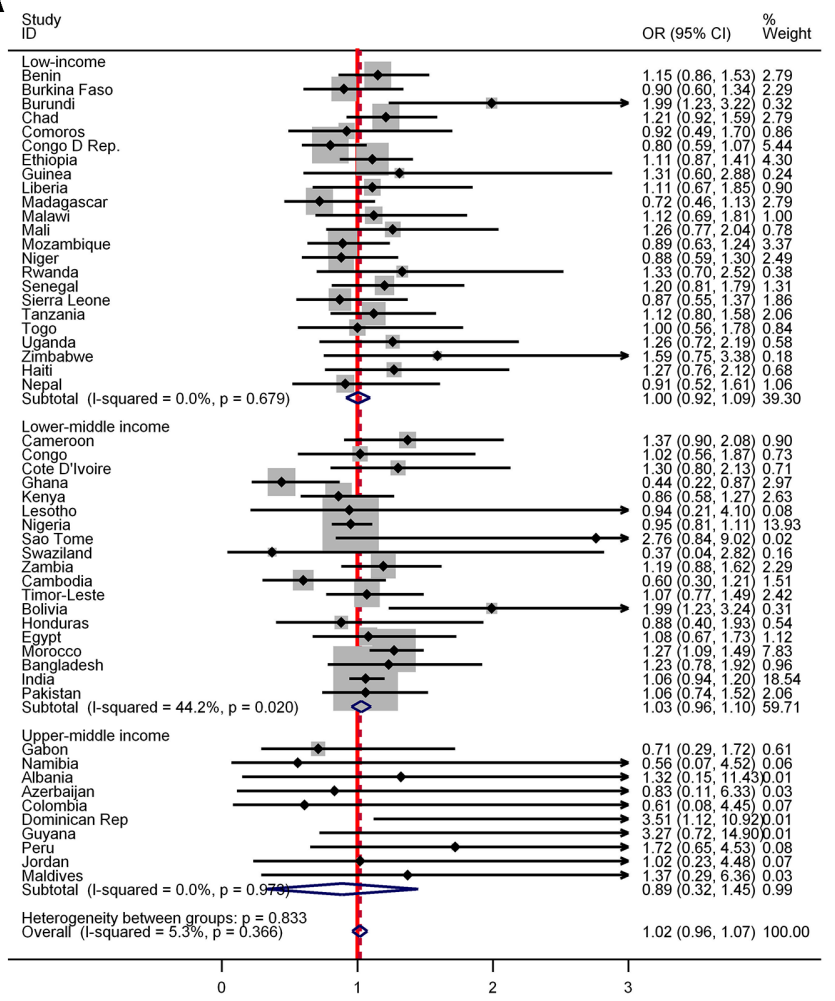

B

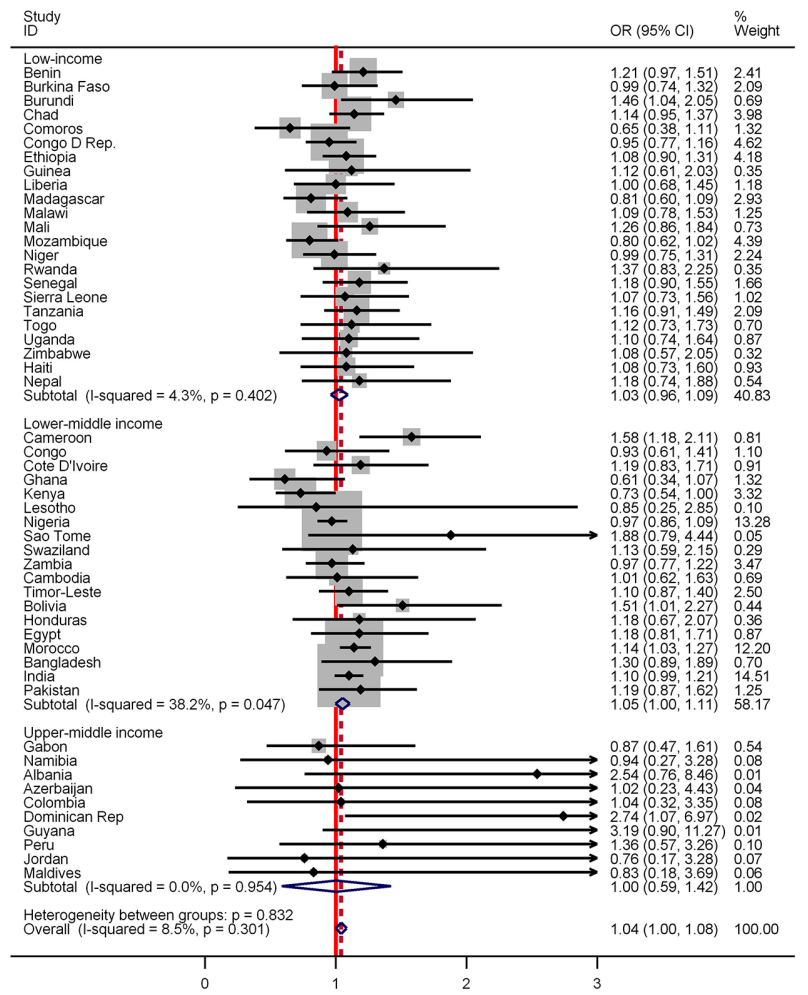

C

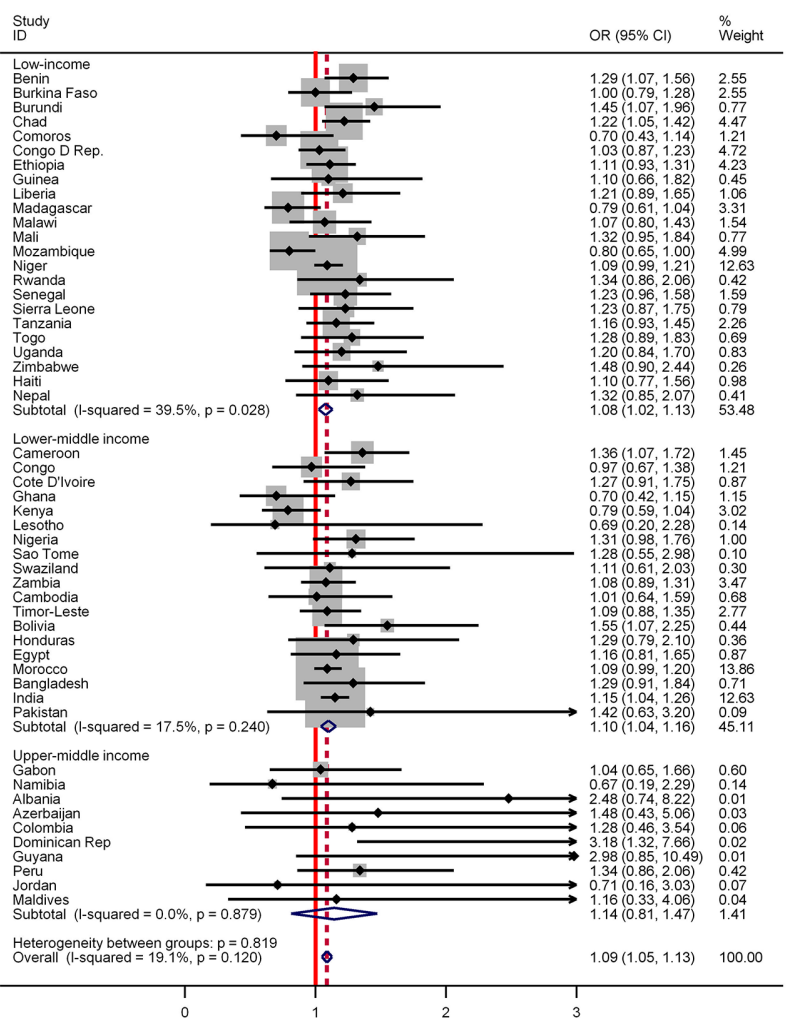

Figure 3 Meta-analysis of the association of low ILWEI with (A) under-5 mortality, (B) infant mortality and (C) neonatal mortality, adjusted for maternal age, wealth quintile, BMI, parity and partner's education by the regions. The associations are presented in ORs and their 95\% Cls for each region in the pooled data. BMI, body mass index; ILWEI, individual-level women's empowerment index.

\section{DISCUSSION}

Our study shows that women's empowerment was low in many of the LMICs studied. Four out of every 10 women did not have autonomy over their own healthcare decision making, only one-fifth had autonomy in all household decision making and just about half 
A
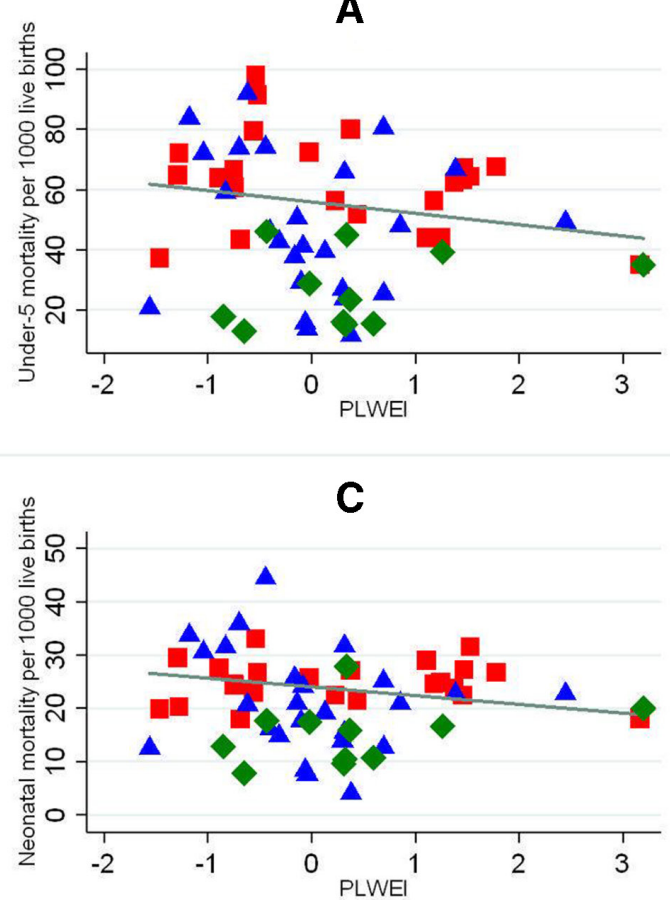

B
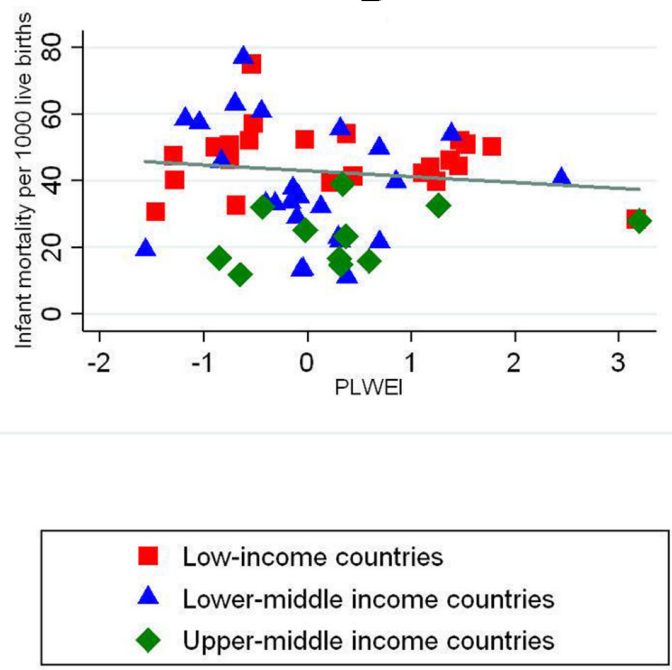

rejected domestic violence against women for any reason. Furthermore, it was found that both ILWEI and PLWEI were associated with higher odds of neonatal, infant and under-5 mortality in LMICs. Our findings from the metaanalysis provide evidence that women's empowerment has the potential to reduce preventable neonatal, infant and under-5 mortality in several LMICs and across the regions.

Women's empowerment is a key global developmental agenda (SDG 5), and while an end in itself, also a means to advance the other SDGs, including those related to child health and survival (SDG 3). Our findings of significant associations between women's empowerment and child survival are consistent with earlier studies, which also found associations of women's empowerment with child health indicators including immunisation completion rate and undernutrition. ${ }^{17}{ }^{18}$ Women's deprivation to access healthcare or their inability to make a decision regarding healthcare can have a direct bearing on their health and their children. In most LMICs, women are the main caregivers of children. Less empowered women may therefore not be able to make key decisions regarding practices affecting their health and their children's, such as compliance with vaccination schedules or the provision of recommended nutrition plans for their children, even if the means were available. Less empowered women are also unlikely to seek appropriate care during childhood illnesses. ${ }^{28}$

Elimination of preventable child deaths in LMICs also requires the availability of reliable information on risk factors and the patterns of distribution across the region to inform evidence-based interventions. Previous studies have mostly explored the immediate causes of child mortality but little empiric information is available on the contribution of underlying causes such as the role of women's empowerment, which are preventable and less cost-intensive. ${ }^{11}$ Our findings suggest that enhancing women's empowerment can avert additional preventable deaths among neonatal, infants and under-5 in LMICs. This can be done by supporting girls' and women's education. It is known that educated women have healthier children who live longer and are more likely to educate their offspring than uneducated women. ${ }^{29}$ Education therefore is one critical women's empowerment tool. In addition, there is the need to provide training for women to acquire the necessary skills and knowledge to manage their own farms, small and large businesses. This will empower them economically to provide for themselves and their families. Such training should take into consideration the social norms and the values of each component of the ILWEI and PLWEI in specific countries. Apart from women's empowerment, other important factors are such as better access to maternal and child healthcare, better access to nutritious food and availability of quality healthcare services to less empowered women, particularly in rural settings is emphasised. These strategies can contribute to improving the health of women and those of their children.

Furthermore, women should be encouraged to participate in local and national governance to ensure that women have a voice in the allocation of resources for the benefit of women and society. The multidimensionality 
of the construct of women's empowerment requires collective efforts of several sectors to provide women with agency and resources and consequently improve neonatal, infant and under-5 survival in LMICs.

Both child survival (SDG 3) and women's empowerment (SDG 5) are important global health and developmental priorities. ${ }^{7-10}$ The SDGs are the most important global agenda driving national policies as well as development assistance for many LMICs for the next one and a half decades. Furthermore, although a number of the SDGs are closely interrelated, there is a relative paucity of empirical evidence that could drive multisectoral interventions towards their attainment. We highlight that there is an important overlap between SDG 3 and SDG 5 that cannot be ignored. ${ }^{21}$ Empowering women can be beneficial to their health and those of their families and can contribute to ending preventable child deaths in LMICs. ${ }^{29} 29{ }^{30}$ While making efforts to empower women, it is important that healthcare systems in LMICs are structured to reach less empowered women's children with life-saving interventions.

Our study has several limitations that we recognise. First, empowerment is a multidimensional construct and there is no known gold standard to measure it, particularly in diverse populations such as LMICs. However, we used multidimensional indicators, which are applicable across a wide range of LMICs to construct women's empowerment indices. Second, the empowerment indices were largely based on self-reported perceptions and recall, and hence could be biassed by social desirability within the individual countries and subject to misclassification. Third, causal inference cannot be emphasised because the data were cross-sectional. Nonetheless, there is evidence that such measures are as accurate as prospective longitudinal surveys. ${ }^{31}$ Fourth, DHSs in LMICs are prone to incomplete or partial reporting of responses. Furthermore, the complex questionnaires used in the DHSs unavoidably allow scope for inconsistent responses to be recorded for different questions. An initial validation study does suggest that the DHSs estimates are accurate. ${ }^{32}$ Despite the above limitations, our study has several strengths. We conducted a meta-analysis on high-quality standardised IPD, which increased the power of our estimates, and further enhanced the conclusiveness of the findings. Additionally, the use of both individual-level and country-level measures of women's empowerment adds to the robustness of the evidence. Furthermore, the data used covered most of the LMICs where data can be accessible $^{57}$ and consequently strengthen the generalisability of our findings. Notwithstanding the limitations of the surveys, they provide the most reliable estimates of child mortality in LMICs. ${ }^{33}$

We conclude that the lack of women's empowerment is associated with excess neonatal, infant and under-5 mortality in LMICs and in all regions. Our study stresses the importance of women's empowerment in accelerating progress towards the attainment of the SDG targets for child survival in LMICs. Holistic, multisectoral and concerted efforts are necessary to eliminate preventable child mortality in LMICs. Such efforts should not only focus on the immediate causes of child mortality but also the underlying causes of the causes, particularly women's empowerment.

\section{Author affiliations}

${ }^{1}$ Department of Population and Health, University of Cape Coast, Cape Coast, Ghana ${ }^{2}$ Unit of Health Sciences, Faculty of Social Sciences, Tampere University, Tampere, Finland

${ }^{3}$ Dalla Lana School of Public Health, University of Toronto, Toronto, Ontario, Canada ${ }^{4}$ Centre for Global Child Health, Sick Kids Foundation, Toronto, Ontario, Canada

Correction notice This article has been corrected since it was published. The article type has been updated.

Acknowledgements Authors' profound gratitude goes to the DHS Program (Macro International Inc.) for granting the permission to use the data for this study.

Contributors DTD and SN: developed the idea and the design of the study; analysed the data, contributed to the data interpretation and wrote the first draft of the manuscript. ZAB: reviewed the manuscript and provided direction for the intellectual content, additional analyses and context. All authors reviewed the draft manuscript and approved the final version for publication.

Funding This research received no specific grant from any funding agency in the public, commercial or not-for-profit sectors.

Map disclaimer The depiction of boundaries on the map(s) in this article do not imply the expression of any opinion whatsoever on the part of BMJ (or any member of its group) concerning the legal status of any country, territory, jurisdiction or area or of its authorities. The map(s) are provided without any warranty of any kind, either express or implied.

\section{Competing interests None declared.}

Patient consent for publication Obtained.

Provenance and peer review Not commissioned; externally peer reviewed.

Data availability statement The data are publicly available at http://dhsprogram. com/Data. Permission is required to use the data from Measure DHS.

Open access This is an open access article distributed in accordance with the Creative Commons Attribution Non Commercial (CC BY-NC 4.0) license, which permits others to distribute, remix, adapt, build upon this work non-commercially, and license their derivative works on different terms, provided the original work is properly cited, appropriate credit is given, any changes made indicated, and the use is non-commercial. See: http://creativecommons.org/licenses/by-nc/4.0/.

\section{REFERENCES}

1 Carlo WA, Goudar SS, Jehan I, et al. Newborn-care training and perinatal mortality in developing countries. $N$ Engl J Med 2010;362:614-23.

2 Bhutta ZA, Black RE, maternal G. Newborn, and child health-so near and yet so far. New Eng J Med 2013;369:2226-35.

3 Lawn JE, Blencowe H, Oza S, et al. Every newborn: progress, priorities, and potential beyond survival. The Lancet 2014;384:189-205.

4 Millennium development goals report, 2015. Available: http://www. un.org/millenniumgoals/2015_MDG_Report/pdf/MDG\%202015\% 20rev\%20\%28July\%201\%29.pdf [Accessed 23 Jan 2017].

5 You D, Hug L, Ejdemyr S, et al. Global, regional, and national levels and trends in under-5 mortality between 1990 and 2015, with scenario-based projections to 2030: a systematic analysis by the un Inter-agency group for child mortality estimation. The Lancet 2015;386:2275-86.

6 Wang H, Bhutta ZA, Coates MM, et al. Global, regional, National, and selected subnational levels of stillbirths, neonatal, infant, and under-5 mortality, 1980-2015: a systematic analysis for the global burden of disease study 2015. The Lancet 2016;388:1725-74.

7 Lim SS, Allen K, Bhutta ZA, et al. Measuring the health-related sustainable development goals in 188 countries: a baseline analysis from the global burden of disease study 2015. The Lancet 2016;388:1813-50.

8 Liu L, Oza S, Hogan D, et al. Global, regional, and national causes of under-5 mortality in 2000-15: an updated systematic analysis 
with implications for the sustainable development goals. The Lancet 2016;388:3027-35.

9 Doku DT, Neupane S. Survival analysis of the association between antenatal care attendance and neonatal mortality in 57 low- and middle-income countries. Int J Epidemiol 2017;46:1668-77.

10 United Nations. Sustainable development goals, 2015. Available: http://www.un.org/sustainabledevelopment/sustainabledevelopment-goals/ [Accessed 24 Jan 2017].

11 Marmot M. Social determinants of health inequalities. The Lancet 2005;365:1099-104.

12 Kabeer N. Resources, agency, achievements: reflections on the measurement of women's empowerment. Dev Change 1999;30:435-64.

13 Lee-Rife SM. Women's empowerment and reproductive experiences over the lifecourse. Soc Sci Med 2010;71:634-42.

14 Mahmud S, Shah NM, Becker S. Measurement of Women's Empowerment in Rural Bangladesh. World Dev 2012;40:610-9.

15 United Nations General Assembly Economic and Social Council. Report of the United nations entity for gender equality and the Empowerment of women on the activities of the United nations trust fund in support of actions to eliminate violence against women, 2016. Available: https://documents-dds ny.un.org/doc/UNDOC/ GEN/N16/447/07/pdf/N1644707.pdf?OpenElement [Accessed 6 Feb 2017].

16 Unite to End Violence Against Women. United Nations Secretary General's Campaign. Available: http://www.un.org/en/women/ endviolence/pdf/VAW.pdf [Accessed 21 Jan 2017].

17 Nilsson M, Griggs D, Visbeck M. Policy: map the interactions between sustainable development goals. Nature 2016;534:320-2.

18 Mistry R, Galal O, Lu M. "Women's autonomy and pregnancy care in rural India: A contextual analysis". Soc Sci Med 2009;69:926-33.

19 Cunningham K, Ruel M, Ferguson E, et al. Women's empowerment and child nutritional status in South Asia: a synthesis of the literature. Matern Child Nutr 2015;11:1-19.

20 Thorpe S, VanderEnde K, Peters C, et al. The influence of women's Empowerment on child immunization coverage in low, LowerMiddle, and Upper-Middle income countries: a systematic review of the literature. Matern Child Health J 2016;20:172-86.

21 James-Hawkins L, Peters C, VanderEnde K, et al. Women's agency and its relationship to current contraceptive use in lower- and middle-income countries: A systematic review of the literature. Glob Public Health 2018;13:843-58.

22 Rosato M, Laverack G, Grabman LH, et al. Community participation: lessons for maternal, newborn, and child health. The Lancet 2008;372:962-71.

23 Ewerling F, Lynch JW, Victora CG, et al. The SWPER index for women's empowerment in Africa: development and validation of an index based on survey data. Lancet Glob Health 2017;5:e916-23.

24 Akseer N, Kamali M, Bakhache N, et al. Status and drivers of maternal, newborn, child and adolescent health in the Islamic world: a comparative analysis. The Lancet 2018;391:1493-512.

25 World bank country and lending groups. Available: http://hdr.undp. org/sites/default/files/hdr2015_technical_notes.pdf [Accessed 21 Jan 2017].

26 United Nations Development Programme. Human development reports. gender development index. Available: http://hdr.undp.org/ en/content/gender-development-index-gdi [Accessed 22 Jan 2017].

27 Guide to demographic and health survey manual. Available: http:// dhsprogram.com/pubs/pdf/DHSG1/Guide_to_DHS_Statistics_ 290ct2012_DHSG1.pdf [Accessed January 2017].

28 Head SK, Yount KM, Sibley LM. Delays in recognition of and Careseeking response to prolonged labor in Bangladesh. Soc Sci Med 2011;72:1157-68.

29 Carr B, Gates MF, Mitchell A, et al. Giving women the power to plan their families. The Lancet 2012;380:80-2.

30 Education for all: achievement and challenges 2000-2015 United nations educational, scientific and cultural organization. UNESCO publishing. Available: http://unesdoc.unesco.org/images/0023/ 002322/232205e.pdf [Accessed 29 Sep 2017].

31 Garenne M, van Ginneken J. Comparison of retrospective surveys with a longitudinal follow-up in Senegal: SFS, DHS and Niakhar. Eur J Population 1994;10:203-21.

32 Macro International Inc. An assessment of the quality of health data in DHS-I surveys. DHS methodological reports No.2. Calverton, Maryland: Macro International Inc, 1993.

33 Neal S. The measurement of neonatal mortality: how reliable is demographic and household survey data? CPC working paper, 2012. Available: http://www.cpc.ac.uk/publications/cpc_working papers/pdf/2012_WP25_The_Measurement_of_Neonatal_Mortality_ Neal.pdf [Accessed 28 Apr 2017]. 